Article

\title{
Gravity Theories with Background Fields and Spacetime Symmetry Breaking
}

\author{
Robert Bluhm \\ Colby College, Department of Physics and Astronomy, Mayflower Hill 5862, Waterville, Maine, 04901, USA; \\ robert.bluhm@colby.edu
}

Received: 25 September 2017; Accepted: 10 October 2017; Published: 16 October 2017

\begin{abstract}
An overview is given of effective gravitational field theories with fixed background fields that break spacetime symmetry. The behavior of the background fields and the types of excitations that can occur depend on whether the symmetry breaking is explicit or spontaneous. For example, when the breaking is spontaneous, the background field is dynamical and massless Nambu-Goldstone and massive Higgs excitations can appear. However, if the breaking is explicit, the background is nondynamical, and in this case additional metric or vierbein excitations occur due to the loss of local symmetry, or these excitations can be replaced by dynamical scalar fields using a Stückelberg approach. The interpretation of Noether identities that must hold in each case differs, depending on the type of symmetry breaking, and this affects the nature of the consistency conditions that must hold. The Noether identities also shed light on why the Stückelberg approach works, and how it is able to restore the broken spacetime symmetry in a theory with explicit breaking.
\end{abstract}

Keywords: gravity; background fields; Lorentz violation; Noether identities.

\section{Introduction}

Some of the most important open questions in physics concern gravity. These include the question of how General Relativity (GR) merges with the Standard Model (SM) of particle interactions in a quantum theory. Additional open questions concern the nature of dark energy and dark matter in gravity theories and cosmology. In many cases, investigations of these questions involve looking at modified gravitational and particle interactions, many of which are described by effective field theories that include fixed background fields. These background fields break spacetime symmetries, such as local Lorentz invariance and diffeomorphism invariance. These breakings can occur spontaneously, where dynamical tensor fields acquire a nonzero vacuum value, or explicitly, when nondynamical background tensors are included directly in the Lagrangian [1].

Examples of effective theories involving background fields in the context of gravity include the Standard-Model Extension (SME) [2-6], Bumblebee models [7-16] or Einstein-Aether models [17,18], Cardinal models [19,20], models with an antisymmetric two-tensor [21,22], Chern-Simons gravity [23,24], massive gravity [25,26], and theories with spacetime-varying couplings [27-31]. In some of these examples, the spacetime symmetry breaking is spontaneous, while in others it is explicit. In certain cases, either type of breaking or even a combination of both types of symmetry breaking can occur [32].

Despite the presence of background fields that break spacetime symmetries, a meaningful physical theory must still be observer independent [2,3]. This means that the choice of spacetime coordinates or local Lorentz bases cannot influence the underlying physics. In the context of a gravity theory, this requires that general coordinate invariance and the passive form of local Lorentz invariance must still hold.

A useful distinction can therefore be made between what are called particle and observer spacetime transformations. Particle transformations act (in an active sense) on physical fields but not 
background fields, while observer transformations act (in a passive sense) on all fields including the background fields. It is the particle symmetries consisting of diffeomorphism invariance and local Lorentz invariance that are broken either spontaneously or explicitly by the presence of background fields, while the mathematical observer symmetries must continue to hold.

If the symmetry breaking is spontaneous, the action describing the theory remains invariant under both the particle and observer transformations when all of the Nambu-Goldstone (NG) and massive Higgs-like excitations are included. However, with explicit breaking, the particle symmetries do not hold. Nonetheless, the observer symmetries must remain mathematical symmetries of the action in order to maintain observer independence. This then sets up a potential conflict between the broken particle symmetries and the unbroken observer symmetries when the symmetry breaking is explicit. However, theories with spontaneous spacetime symmetry breaking do not encounter such conflicts [5].

In the context of a gravitational theory, which must respect geometrical identities such as the Bianchi identities, the conflicts that arise with explicit breaking can lead to theoretical inconsistency unless certain conditions hold. One approach for obtaining useful consistency conditions is to look at the mathematical Noether identities associated with the observer invariances [33]. Using these identities, the question of whether a particular theory is consistent or not can then be examined.

In this overview, a general treatment is used to examine different features and behaviors that can arise in gravity theories that contain background fields, including their dependence on whether the spacetime symmetry breaking is spontaneous or explicit. First, in the next section, the properties of the background fields are examined for the two types of symmetry breaking. Next, in Section 3, the different types of excitations that can occur in conjunction with the symmetry breaking are investigated and discussed. These include massless Nambu-Goldstone (NG) and massive Higgs-like modes in the case of spontaneous breaking, or additional metric modes or Stückelberg fields in the case of explicit breaking. Section 4 looks at the Noether identities that hold and how their interpretation depends on the form of the symmetry breaking. A summary and conclusions are given in Section 5 .

It is important to keep in mind that a major component of the research effort devoted to testing spacetime symmetries consists of experimental tests of local Lorentz symmetry and GR [34-51]. The SME is widely used as the phenomenological framework for these tests, and the sensitivities to Lorentz violation are expressed as experimental bounds on the SME coefficients [52]. This overview will not discuss experimental tests of Lorentz symmetry. However, the question of how background fields are interpreted depending on the type of symmetry breaking is relevant to the SME. For example, in the SME restricted to Minkowski spacetime, the coefficients for Lorentz violation can be treated as fixed nondynamical background fields that explicitly break Lorentz symmetry. However, in the gravity sector of the SME [53-63] more caution is usually used in the case of explicit breaking. Typically, the pure-gravity sector of the SME assumes the background SME coefficients are dynamical vacuum solutions and that the NG and Higgs modes must be accounted for in order to avoid potential inconsistency issues.

\section{Background Fields}

A variety of background fields are considered in effective gravitational field theories. These include fixed nondynamical background scalar or tensor fields, which explicitly break diffeomorphism invariance and local Lorentz invariance. Alternatively, dynamical background fields can arise as vacuum expectation values if the symmetry breaking is spontaneous.

To consider these types of models in a general way, and to examine the differences between explicit and spontaneous spacetime symmetry breaking, let $\bar{k}_{\lambda \mu \nu} \ldots$ denote a generic background field. Consider it as a fixed scalar or tensor with an unspecified number of components. An effective gravitational theory containing such a background also includes interactions with other fields that are fully dynamical, including the metric, $g_{\mu v}$, and conventional matter fields. The latter are denoted generically as $f^{\psi}$, where $\psi$ is a collective label for the all of the matter-field tensor indices. Assuming 
an Einstein-Hilbert form for the pure-gravity sector, and using units with $8 \pi G=1$, the Lagrangian for a theory of this form can be written as

$$
S=\int d^{4} x \sqrt{-g}\left[\frac{1}{2} R+\mathcal{L}\left(g_{\mu v}, f^{\psi}, \bar{k}_{\lambda \mu \nu \cdots}\right)\right] .
$$

The equations of motion are obtained by varying $S$ with respect to the dynamical fields. This yields the Einstein equations, $G^{\mu v}=T^{\mu v}$, when variations with respect to $g_{\mu v}$ are performed. Variations with respect to the matter fields $f^{\psi}$ yield the Euler-Lagrange equations for $f^{\psi}$, which can be denoted generically as $\frac{\delta \mathcal{L}}{\delta f^{\psi}}=0$. These equations typically include partial derivative contributions. For example, if $\mathcal{L}$ depends on both $f^{\psi}$ and $D_{\mu} f^{\psi}$, then the Euler-Lagrange expression is

$$
\frac{\delta \mathcal{L}}{\delta f^{\psi}} \equiv-D_{\mu}\left(\frac{\partial \mathcal{L}}{\partial D_{\mu} f^{\psi}}\right)+\frac{\partial \mathcal{L}}{\partial f^{\psi}}
$$

where $D_{\mu}$ is a covariant derivative.

The question of whether the background field obeys Euler-Lagrange equations or not depends on the type of symmetry breaking. With explicit breaking, the background is nondynamical, and therefore Euler-Lagrange equations need not hold. Thus, in general

$$
\frac{\delta \mathcal{L}}{\delta \bar{k}_{\lambda \mu \nu \cdots}} \neq 0
$$

However, if the symmetry breaking is spontaneous, then the background $\bar{k}_{\lambda \mu \nu \ldots}$ arises as a vacuum

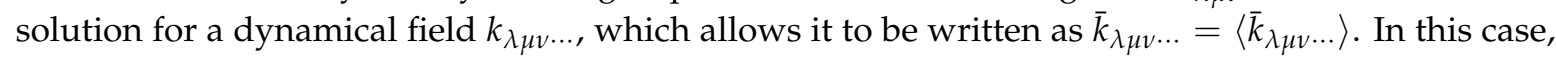
$\bar{k}_{\lambda \mu \nu \ldots}$ is a solution of the vacuum Euler-Lagrange equations,

$$
\left(\frac{\delta \mathcal{L}}{\delta \bar{k}_{\lambda \mu v \cdots}}\right)_{\text {vacuum }}=0 .
$$

Away from the vacuum solution, the dynamical field $k_{\lambda \mu v} \ldots$ has excitations in the form of massless NG and massive Higgs-like modes. When these are included in the effective theory, particle diffeomorphism and local Lorentz invariance still hold, and $k_{\lambda \mu \nu} \ldots$ is a solution of its Euler-Lagrange equations.

\subsection{Diffeomorphism Breaking}

Since the metric and conventional matter fields are fully dynamical, they transform under particle diffeomorphisms in the usual way, with infinitesimal changes given by Lie derivatives defined with respect to a spacetime vector $\xi^{\mu}$. For example, the metric transforms as

$$
g_{\mu v} \rightarrow g_{\mu v}+\mathcal{L}_{\xi} g_{\mu v}=g_{\mu \nu}+D_{\mu} \xi_{v}+D_{\nu} \xi_{\mu}
$$

while the matter fields transform as

$$
f^{\psi} \rightarrow f^{\psi}+\mathcal{L}_{\xi} f^{\psi}
$$

However, the background field breaks particle diffeomorphisms and remains fixed under these transformations, obeying

$$
\bar{k}_{\lambda \mu \nu \cdots} \stackrel{\text { particle }}{\longrightarrow} \bar{k}_{\lambda \mu \nu \ldots}
$$

At the same time, to be physically viable, the theory must be observer independent. This requires that the action remains invariant under general coordinate transformations. For infinitesimal coordinate transformations, $x^{\mu} \rightarrow x^{\mu}-\xi^{\mu}$, defined using vectors, $-\xi^{\mu}$, in the inverse direction, all of the tensor fields in the theory transform mathematically with changes given by Lie derivatives. For this 
reason, these transformations are referred to as observer diffeomorphisms. The metric and conventional matter fields transform the same way under these infinitesimal coordinate transformations as they do under particle diffeomorphisms. Note, however, that under these observer transformations, the background field also transforms, obeying

$$
\bar{k}_{\lambda \mu \nu \ldots} \stackrel{\text { observer }}{\longrightarrow} \bar{k}_{\lambda \mu \nu \cdots}+\mathcal{L}_{\xi} \bar{k}_{\lambda \mu \nu \ldots}
$$

If the diffeomorphism breaking is explicit, then the action is not invariant, and $\delta S \neq 0$ under particle diffeomorphisms. Nonetheless, the action is still required to obey $\delta S=0$ mathematically under the observer diffeomorphisms in order to maintain observer independence. It is in this way that the explicit breaking of diffeomorphisms while maintaining observer independence can lead to a potential conflict. To be fully consistent, a theory must resolve or evade this conflict.

With explicit diffeomorphism breaking, four local gauge invariances associated with the local vectors $\xi^{\mu}$ do not occur. As a result, there are up to four additional degrees of freedom in the metric compared to GR, which can therefore modify gravitational interactions. These extra metric modes can also give rise to ghosts, which is therefore an important consideration in theories with explicit diffeomorphism breaking.

In many theories with explicit diffeomorphism breaking, a Stückelberg approach is used [64]. In this approach, the background $\bar{k}_{\lambda \mu \nu \ldots}$ is rewritten in terms of four dynamical scalars, $\phi^{A}$, labeled with an index $A=0,1,2,3$. The replacement is given as

$$
\bar{k}_{\lambda \mu \nu \cdots}(x)=\partial_{\lambda} \phi^{A} \partial_{\mu} \phi^{B} \partial_{\nu} \phi^{C} \cdots \bar{k}_{A B C \cdots}(\phi) .
$$

The four scalars transform under particle diffeomorphisms, and the substitution of (9) into the action $S$ is sufficient to restore particle diffeomorphism invariance. As a result, four degrees of freedom in the metric can again be treated as gauge degrees of freedom. However, the theory still has up to four additional degrees of freedom, in comparison to GR, due to the added Stückelberg fields.

With spontaneous diffeomorphism breaking, the number of degrees of freedom in the metric is similar to GR. This is because particle diffeomorphism invariance still holds when the NG modes are included in the action. Thus, there are four local gauge degrees of freedom that can be used to eliminate four degrees of freedom in the metric.

\subsection{Local Lorentz Symmetry Breaking}

To reveal the local Lorentz invariance, a vierbein formalism can be used. In this case, the metric is replaced by a vierbein $e_{a}{ }^{\mu}$, where the defining relation is

$$
g_{\mu v}=e_{\mu}{ }^{a} e_{v}^{b} \eta_{a b}
$$

Here, Greek indices are used for components defined on the spacetime manifold, while Latin indices denote components defined with respect to a local Lorentz frame.

Again, a distinction can be made between particle and observer transformations. Under particle local Lorentz transformations, which depend on six antisymmetric parameters $\epsilon_{a b}$, the vierbein transforms as a local vector,

$$
e_{\mu}^{a} \rightarrow e_{\mu}^{a}+\epsilon^{a}{ }_{b} e_{\mu}^{b} .
$$

The matter fields $f^{\psi}$ have components with respect to the local basis, which can be denoted generically as $f^{y}$. These transform as irreducible representations of the local Lorentz group, which have the form

$$
f^{y} \rightarrow f^{y}+\frac{1}{2} \epsilon^{a b}\left(X_{[a b]}\right)_{x}^{y} f^{x} .
$$

Since the background field is fixed, both the components $\bar{k}_{\lambda \mu \nu \ldots}$ defined with respect to the spacetime coordinate frame and $\bar{k}_{a b c \ldots}$. defined with respect to the local Lorentz frame remain 
unchanged under particle spacetime transformations. Since the frames themselves do not change either under particle transformations, the background components are therefore connected by a fixed background vierbein, denoted as $\bar{e}_{a}{ }^{\mu}$. The defining relation for the background vierbein is

$$
\bar{k}_{\lambda \mu \nu \cdots}=\bar{e}_{\lambda}^{a} \bar{e}_{\mu}^{b} \bar{e}_{v}^{c} \cdots \bar{k}_{a b c \cdots}
$$

where each quantity in this expression remains fixed under both particle diffeomorphisms and local Lorentz transformations.

In a vierbein formalism, the action replacing (1) can then be written as

$$
S=\int d^{4} x e\left[\frac{1}{2} R+\mathcal{L}\left(e_{\mu}{ }^{a}, \bar{e}_{\mu}{ }^{a}, f^{y}, \bar{k}_{a b c \cdots}\right)\right],
$$

where $e$ is the determinant of the vierbein. The action in this case depends on the dynamical vierbein and conventional matter fields as well as the background $\bar{k}_{a b c \ldots}$ and fixed vierbein $\bar{e}_{\mu}{ }^{a}$.

If the symmetry breaking is explicit, the background field and the background vierbein are nondynamical, and the action is not invariant under particle diffeomorphisms and local Lorentz transformations. However, in order to maintain observer independence, the action must be mathematically invariant under observer local Lorentz transformations, consisting of changes of the local Lorentz bases, as well as observer diffeomorphisms. In this case, all of the fields, including $\bar{k}_{a b c \ldots}$ and $\bar{e}_{a}{ }^{\mu}$, transform so as to keep $S$ unchanged. The combination of broken particle local Lorentz invariance with unbroken observer local Lorentz symmetry can lead to potential conflicts similar to those that occur with explicit diffeomorphism breaking. Theoretical consistency requires that these conflicts must be avoided as well.

However, if the symmetry breaking is spontaneous, then both the background $\bar{k}_{a b c \ldots}$ and the background vierbein $\bar{e}_{a}{ }^{\mu}$ arise dynamically as vacuum expectation values. When the NG modes for both the broken diffeomorphism invariance and local Lorentz invariance are included in the action, the symmetry of $S$ under both sets of transformations is restored.

\section{Excitations}

Effective gravitational field theories with background fields have excitations that depend on the form of the symmetry breaking.

A theory with spontaneous diffeomorphism and local Lorentz violation has excitations that occur as massless NG and massive Higgs excitations. The question of whether a gravitational Higgs mechanism can occur becomes relevant as well.

In contrast, in a theory with explicit diffeomorphism and local Lorentz breaking, the background field is nondynamical and does not have excitations. The fact that the background is not able to have backreactions and provides a structure with "prior geometry" is very different from GR and theories with spontaneous spacetime symmetry breaking. However, the loss of local symmetry in theories with explicit breaking does give rise to additional degrees of freedom in the metric, and these additional excitations lead to modified gravitational interactions.

In theories with explicit breaking, a Stückelberg approach is often used. In this case, the multi-component background is replaced by a function, including derivatives, of four dynamical scalar fields, and the local spacetime symmetry is restored. Excitations of the Stückelberg fields have the form of NG excitations. Thus, the question of how these excitations compare with the NG excitations in a theory with spontaneous breaking is pertinent.

\subsection{NG Modes}

Typically, a potential term $V\left(g_{\mu v}, k_{\lambda \mu \nu \ldots}\right)$ in the Lagrangian induces spontaneous spacetime symmetry breaking, where the potential is formed from scalar combinations of $g_{\mu \nu}$ and $k_{\lambda \mu \nu \ldots}$ and possibly their derivatives and other fields as well. The spontaneous diffeomorphism breaking occurs 
when a nonzero solution $\bar{k}_{\lambda \mu \nu \ldots}$ and $\left\langle g_{\mu \nu}\right\rangle$ causes the potential to be at a minimum obeying $V^{\prime}=0$. Massless NG modes then occur as excitations about the vacuum solution that stay in the minimum (still obeying $V^{\prime}=0$ ), while massive Higgs excitations are solutions that do not stay in the minimum (with $V^{\prime} \neq 0$ ).

The NG modes are generated by the broken symmetry transformations. With broken diffeomorphims, they have the form of infinitesimal Lie derivatives, where the four parameters $\xi^{\mu}$ become the NG degrees of freedom. The excitations can then be written at leading order as

$$
k_{\lambda \mu \nu \cdots} \simeq \bar{k}_{\lambda \mu \nu \cdots}+\left(D_{\lambda} \xi^{\alpha}\right) \bar{k}_{\alpha \mu \nu \cdots}+\left(D_{\mu} \xi^{\alpha}\right) \bar{k}_{\mu \alpha \nu \cdots}+\cdots+\xi^{\alpha} D_{\alpha} \bar{k}_{\alpha \mu \nu \cdots}+\left(\delta k_{\lambda \mu \nu \cdots}\right)_{\text {massive }}
$$

where $\left(\delta k_{\lambda \mu v \ldots}\right)_{\text {massive }}$ denotes the massive Higgs modes. While there are only four NG modes associated with diffeomorphism breaking, the number of massive modes depends on the type of tensor, the potential $V$, and the kinetic terms for $k_{\lambda \mu v} \ldots$. The question of whether ghost modes exist depends on these features as well.

To generate the NG modes for the broken local Lorentz transformations, infinitesimal excitations having the form of broken Lorentz transformations around the vacuum solution in a vierbein formalism can be used. In this case, there are six NG excitations, which can be written in terms of $\epsilon_{a b}$ as

$$
k_{a b c \ldots} \simeq \bar{k}_{a b c \ldots}+\epsilon_{a}^{j} \bar{k}_{j b c \ldots}+\epsilon_{b}^{j} \bar{k}_{a j c \ldots}+\cdots+\left(\delta k_{a b c \ldots}\right)_{\text {massive }}
$$

where $\left(\delta k_{a b c \ldots}\right)$ massive are the components of the massive excitations defined with respect to the local Lorentz frame.

In theories with spontaneous spacetime symmetry breaking, the NG modes can be interpreted in some cases as known gauge fields, such as photons or gravitons $[10,11,19,20,65,66]$. Alternatively, the NG modes can be gauged into the vierbein, which modifies the gravitational interactions. If a Riemann-Cartan geometry is considered, theories with a Higgs mechanism that gives rise to mass terms for the spin connection become possible $[10,11]$. However, finding models that are free of ghosts remains elusive.

\subsection{Stückelberg Fields}

If a Stückelberg approach is used in a theory with explicit diffeomorphism breaking, the nondynamical background is replaced by four dynamical scalars as shown in (9), which restores the local diffeomorphism invariance. The Stückelberg version is dynamically equivalent to the original explicit-breaking form, since imposing gauge-fixing conditions on the scalars, $\phi^{A}=\delta_{\mu}^{A} x^{\mu}$, reduces the expression on the right-hand side in (9) back to $\bar{k}_{\lambda \mu v} \ldots$ and leaves the metric with four additional degrees of freedom.

To restore diffeomorphism invariance, the excitations in the Stückelberg scalars have the form of NG modes. Writing the excitations as

$$
\phi^{A}=\delta_{\mu}^{A}\left(x^{\mu}+\xi^{\mu}\right)
$$

and substituting them into the expression in (9) gives the leading order that

$$
\partial_{\lambda} \phi^{A} \partial_{\mu} \phi^{B} \partial_{\nu} \phi^{C} \ldots \bar{k}_{A B C} \ldots(\phi) \simeq \bar{k}_{\lambda \mu \nu \ldots}+\left(D_{\lambda} \xi^{\alpha}\right) \bar{k}_{\alpha \mu \nu \ldots}+\left(D_{\mu} \xi^{\alpha}\right) \bar{k}_{\mu \alpha v \ldots}+\cdots+\xi^{\alpha} D_{\alpha} \bar{k}_{\alpha \mu \nu \ldots}
$$

This has the same form as the NG excitations about the background $\bar{k}_{\lambda \mu \nu \ldots}$ in a corresponding theory with spontaneous breaking, as given in (15), but where there are no massive excitations.

An explicit-breaking theory in a Stückelberg description can be viewed as a theory with spontaneous diffeomorphism breaking, but where it is the scalar fields that acquire vacuum values of the form $\phi^{A}=\delta_{\mu}^{A} x^{\mu}$. While these vacuum values replicate the original background $\bar{k}_{\lambda \mu \nu \cdots}$ as it appears in the Lagrangian, there are still only four degrees of freedom in the explicit-breaking case. 
This is, in general, not sufficient to provide a vacuum solution for the multi-component background tensor $\bar{k}_{\lambda \mu \nu} \ldots$, which must satisfy Euler-Lagrange equations for all of its components if it is to be fully dynamical. Thus, instead, (3) continues to hold for the explicit-breaking theory, and $\bar{k}_{\lambda \mu v \ldots}$ remains nondynamical.

\section{Noether Identities}

Field theories with local symmetries obey Noether's second theorem $[67,68]$, which states that off-shell identities relating the Euler-Lagrange expressions for the dynamical fields must hold. The main consequence of the Noether identities is that not all of the equations of motion are independent when there are local symmetries.

For example, in GR with matter fields $f^{\psi}$, the Noether identities that result from diffeomorphism invariance have the form

$$
D_{\mu}\left(G^{\mu v}-T^{\mu v}\right)+\frac{\delta \mathcal{L}}{\delta f^{\psi}} \gamma^{\psi v}+D_{\mu}\left(\frac{\delta \mathcal{L}}{\delta f^{\psi}} \gamma^{\psi \mu \nu}\right)=0,
$$

The coefficients $\gamma^{\psi v}$ and $\gamma^{\psi \mu v}$ denote functions of the field components, where their specific form depends on the theory, while $\left(G^{\mu v}-T^{\mu v}\right)$ and $\frac{\delta \mathcal{L}}{\delta f^{\psi}}$ are the Euler-Lagrange expressions for the metric and matter fields, respectively. What this identity says is that four of the dynamical equations of motion are not independent when there is local diffeomorphism invariance. Alternatively, when this identity is combined with the contracted Bianchi identity, which states that $D_{\mu} G^{\mu v}=0$, it shows that covariant energy-momentum conservation, $D_{\mu} T^{\mu v}=0$, must automatically hold when the dynamical matter fields are on-shell obeying $\frac{\delta \mathcal{L}}{\delta f^{\psi}}=0$.

In a vierbein description, Noether identities resulting from local Lorentz invariance hold as well. Using a vierbein description in GR with matter fields $f^{\psi}$, the resulting Noether identities are

$$
\left(G^{\mu v}-T^{\mu v}\right)\left(e_{\mu a} e_{v b}-e_{\mu b} e_{v a}\right)+\frac{1}{2} \frac{\delta \mathcal{L}}{\delta f^{y}}\left(X_{[a b]}\right)^{y}{ }_{x} f^{x}=0 .
$$

In this case, since $G^{\mu v}=G^{v \mu}$ holds for the Einstein tensor, the result of this identity is that the energy-momentum tensor in the vierbein description must also be symmetric when the matter fields are on shell.

While effective gravitational theories with background fields break diffeomorphism and local Lorentz invariance either explicitly or spontaneously, it may seem that there are no Noether identities that apply in these theories. However, if the theory is to remain observer independent, the action must still be mathematically invariant under the observer spacetime transformations. Applying the observer spacetime transformations and imposing $\delta S=0$ therefore results in Noether identities that must hold even when there is a background field. Under the observer transformations, the background field transforms along with the metric and matter fields, which can yield Noether identities for both observer diffeomorphisms and local Lorentz transformations [33].

For example, performing observer diffeomorphisms on the action in (1) and requiring that it be observer independent results in Noether identities of the form

$$
D_{\mu}\left(G^{\mu v}-T^{\mu v}\right)+\frac{\delta \mathcal{L}}{\delta f^{\psi}} \gamma^{\psi v}+D_{\mu}\left(\frac{\delta \mathcal{L}}{\delta f^{\psi}} \gamma^{\psi \mu \nu}\right)+\frac{\delta \mathcal{L}}{\delta \bar{k}_{\alpha \beta \gamma \ldots}} \lambda_{\alpha \beta \gamma \ldots}^{v}+D_{\mu}\left(\frac{\delta \mathcal{L}}{\delta \bar{k}_{\alpha \beta \gamma \cdots}} \lambda_{\alpha \beta \gamma \ldots}^{\mu v}\right)=0 .
$$

In this case, $\gamma^{\psi v}, \gamma^{\psi \mu v}, \lambda_{\alpha \beta \gamma \ldots}^{v}$ and $\lambda_{\alpha \beta \gamma \ldots}^{\mu \nu}$ all denote coefficients that are functions of the field components. Notice in this case that Euler-Lagrange expressions for the background, $\frac{\delta \mathcal{L}}{\delta \bar{k}_{\alpha \beta \gamma} \ldots}$, appear in this identity. As a result of this, the interpretation of the Noether identies when there is a background field present depends on whether the symmetry breaking is explicit or spontaneous. 
For the case of explicit breaking, the background $\bar{k}_{\lambda \mu \nu \ldots}$ is nondynamical and the Euler-Lagrange equation for it need not vanish, as indicated in (3). Thus, when the metric and conventional matter fields are on shell, theoretical consistency requires that the following equation must hold:

$$
\frac{\delta \mathcal{L}}{\delta \bar{k}_{\alpha \beta \gamma \cdots}} \lambda_{\alpha \beta \gamma \ldots}^{v}+D_{\mu}\left(\frac{\delta \mathcal{L}}{\delta \bar{k}_{\alpha \beta \gamma \ldots}} \lambda_{\alpha \beta \gamma \ldots}^{\mu v}\right)=0
$$

This results in a different interpretation from GR, since it is no longer an option to set the Euler-Lagrange expressions for $\bar{k}_{\lambda \mu \nu \ldots}$ to zero. Instead, it is the four additional metric modes that exist as a result of the symmetry breaking that must satisfy this equation. In some theories, the couplings between the additional metric modes and the background are insufficient to allow the conditions in (22) to hold. For example, if a particular ansatz form of the metric is chosen that does not include any of the needed additional modes, then some backgrounds can become incompatible with the Noether identities.

However, if the symmetry breaking is spontaneous, then $\bar{k}_{\lambda \mu v} \ldots$ is a dynamical vacuum solution and Euler-Lagrange equations hold, as in (4). If excitations are included as in (15), then the Euler-Lagrange equations for the tensor $k_{\lambda \mu \nu \cdots}$ (including the NG and massive excitations) hold. Thus, with spontaneous diffeomorphism breaking, the interpretation of the Noether identities is the same as in GR. Four of the equations of motion are not dynamically independent, and covariant energy-momentum conservation holds when all of the dynamical fields are on shell.

If a vierbein formalism is used, the Noether identities resulting from observer local Lorentz invariance can be obtained. In this case, the action is given in (14), which depends on both the background field and a background vierbein. Requiring that $S$ be unchanged under observer local Lorentz transformations gives six Noether identities, which have the form

$$
\begin{gathered}
\left(G^{\mu v}-T^{\mu v}\right)\left(e_{\mu a} e_{v b}-e_{\mu b} e_{v a}\right)+\frac{1}{2} \frac{\delta \mathcal{L}}{\delta f^{y}}\left(X_{[a b]}\right)^{y}{ }_{x} f^{x}+\left(\frac{\delta \mathcal{L}}{\delta \bar{e}_{\mu}{ }^{a}} \bar{e}_{\mu b}-\frac{\delta \mathcal{L}}{\left.\delta \bar{e}_{\mu}{ }^{b} \bar{e}_{\mu a}\right)}\right. \\
+\frac{\delta \mathcal{L}}{\delta \bar{k}_{c d e \ldots}}\left[\left(\eta_{a c} \bar{k}_{b d e \ldots}-\eta_{b c} \bar{k}_{a d e \ldots}\right)+\left(\eta_{a d} \bar{k}_{c b e \ldots}-\eta_{b d} \bar{k}_{c a e \ldots}\right)\right. \\
\left.+\left(\eta_{a c} \bar{k}_{c d b \ldots}-\eta_{b c} \bar{k}_{c d a \ldots}\right)+\cdots\right]=0 .
\end{gathered}
$$

Again, the interpretation of these identities depends on the type of symmetry breaking.

With explicit breaking of local Lorentz invariance, the background field and the background vierbein are nondynamical and therefore in general obey the relations

$$
\frac{\delta \mathcal{L}}{\delta \bar{e}_{\mu}{ }^{a}} \neq 0, \quad \frac{\delta \mathcal{L}}{\delta \bar{k}_{a b c \cdots}} \neq 0 .
$$

Thus, when the matter fields are on shell and the symmetry of the Einstein tensor is used, it follows that the energy-momentum tensor in the vierbein description is symmetric only if the remaining terms in (23) combine to give zero. With explicit Lorentz breaking, the vierbein has six additional degrees of freedom due to the loss of the local symmetry. It is these degrees of freedom that must make the remaining terms in (23) vanish in order for $T^{\mu v}$ to be symmetric.

On the other hand, if the breaking of local Lorentz invariance is spontaneous, then both $\bar{e}_{\mu}{ }^{a}$ and $\bar{k}_{a b c \ldots}$ are dynamical vacuum solutions. Therefore, the Euler-Lagrange equations for the vacuum hold, and these equations continue to hold when excitations are included. The result in this case is the same as in GR. When the all the dynamical equations of motion hold, $T^{\mu v}$ is automatically symmetric.

As described above, the Stückelberg formalism allows an explicit-breaking theory with a nondynamical background $\bar{k}_{\lambda \mu \nu \ldots}$ to be reinterpreted as a dynamical theory with four additional scalars $\phi^{A}$. The Stückelberg approach is often referred to as a trick, since it restores the symmetry in a theory where it is initially explicitly broken, and it makes the theory dynamical. Some insight into why this trick works can be obtained by examining the Noether identities that hold with scalar fields. 
If the Stückelberg substitution (9) is made in the action in (1), the result is a new action that depends on the fields $g_{\mu v}, f^{\psi}$, and $\phi^{A}$. The Noether identities stemming from diffeomorphism transformations in this case are

$$
D_{\mu}\left(G^{\mu v}-T^{\mu v}\right)+\frac{\delta \mathcal{L}}{\delta f^{\psi}} \gamma^{\psi v}+D_{\mu}\left(\frac{\delta \mathcal{L}}{\delta f^{\psi}} \gamma^{\psi \mu v}\right)+\left(-D_{\mu} \frac{\partial \mathcal{L}}{\partial \partial_{\mu} \phi^{A}}+\frac{\partial \mathcal{L}}{\partial \phi^{A}}\right) \partial_{\nu} \phi^{A}=0 .
$$

When the metric and matter fields are put on shell, and assuming the derivatives $\partial_{\nu} \phi^{A}$ are linearly independent, the resulting conditions that must hold are

$$
-D_{\mu} \frac{\partial \mathcal{L}}{\partial \partial_{\mu} \phi^{A}}+\frac{\partial \mathcal{L}}{\partial \phi^{A}}=0
$$

These have the form of the Euler-Lagrange equations of motion for the scalars $\phi^{A}$.

It is important to realize that the Noether identities in (25) and the conditions in (26) that follow from them can be obtained in two different ways. In the first, the scalars $\phi^{A}$ are treated as fixed nondynamical background fields that explicitly break diffeomorphism invariance. The substitution (9) in this case replaces the nondynamical background $\bar{k}_{\lambda \mu \nu \ldots}$ by derivatives of nondynamical scalars $\phi^{A}$. The Noether identities in (25) follow, in this case, from imposing the requirement of observer independence and using observer diffeomorphism transformations. However, the same identities in (25) follow using the Stückelberg trick, where the scalars $\phi^{A}$ in this case are treated as dynamical fields. It is unbroken diffeomorphism invariance that gives rise to the Noether identities in (25) in this approach.

Thus, using either nondynamical or dynamical scalars $\phi^{A}$, the result of the Noether identities is that the Euler-Lagrange equations in (26) must hold. It is this fact that enables the Stückelberg approach to work. Starting with a theory with explicit breaking and a nondynamical background, the replacement in (9) is made using fixed nondynamical scalars. The consistency of the explicit-breaking theory requires that the conditions stemming from the Noether identities must hold. However, these have the form of the Euler-L-agrange equations for the scalars. Letting the scalars be dynamical and restoring diffeomorphism invariance is then possible because the Euler-Lagrange equations for the dynamical scalars are already imposed.

It is important to note as well that in the first case where the scalars $\phi^{A}$ are nondynamical, the Euler-Lagrange equations in (26) must be satisfied by the additional metric modes that occur due to the lose of local symmetry. In contrast, in the Stückelberg approach, where the scalars are dynamical and the metric can be gauge fixed, it is then the scalars themselves that must satisfy their own Euler-Lagrange equations, as expected for fields that are dynamical.

\section{Summary and Conclusions}

Effective gravitational field theories with background fields are used in a variety of investigations looking at possible modifications of gravity, quantum gravity effects, and phenomenological effects of spacetime symmetry breaking. The presence of a background field breaks diffeomorphism and local Lorentz invariance either explicitly or spontaneously, and the behavior and interpretation of the background field depends on which type of breaking occurs.

With explicit breaking, the background is nondynamical, and it does not obey Euler-Lagrange equations of motion. Noether identities in this case are obtained by imposing observer independence. It is the additional metric modes that result from the absence of local symmetry that must satisfy the Noether identities.

In contrast, with spontaneous breaking, the background is dynamical and it obeys Euler-Lagrange equations either as a vacuum solution or when the NG and massive Higgs modes are included. Noether identities follow in this case from the unbroken local symmetry, and their interpretation is similar to GR. 
A Stückelberg approach can be used to turn a theory with explicit breaking and a nondynamical background into an equivalent theory with four dynamical scalars where the symmetry is restored. The Stückelberg excitations are NG modes about vacuum solutions for the four scalars. The original fixed background $\bar{k}_{\lambda \mu \nu \ldots}$ remains nondynamical and does not satisfy its Euler-Lagrange equations. Instead, it is the scalar field Euler-Lagrange equations that hold. The fact that these equations hold regardless of whether the scalars are dynamical or nondynamical is a key feature that permits the Stückelberg approach to work.

Conflicts of Interest: The author declares no conflicts of interest.

\section{References}

1. Bluhm, R. Explicit versus spontaneous diffeomorphism breaking in gravity. Phys. Rev. D 2015, 91, 065034.

2. Colladay, D.; Kostelecký, V.A. CPT violation and the standard model. Phys. Rev. D 1997, 55, 6760.

3. Colladay, D.; Kostelecký, V.A. Lorentz-violating extension of the standard model. Phys. Rev. D 1998, 58, 116002.

4. Kostelecký, V.A.; Lehnert, R. Stability, causality, and Lorentz and CPT violation. Phys. Rev. D 2001, 63, 065008.

5. Kostelecký, V.A. Gravity, Lorentz violation, and the standard model. Phys. Rev. D 2004, 69, 105009.

6. Bluhm, R. Overview of the SME: Implications and Phenomenology of Lorentz Violation. In Special Relativity: Will It Survive the Next 101 Years? Ehlers, J., Lämmerzahl, C., Eds.; Springer: Berlin, Germany, 2006.

7. Kostelecký, V.A.; Samuel, S. Gravitational phenomenology in higher dimensional theories and strings. Phys. Rev. D 1989, 40, 1886.

8. Kostelecký, V.A.; Samuel, S. Spontaneous breaking of Lorentz symmetry in string theory. Phys. Rev. D 1989, 39,683 .

9. Kostelecký, V.A.; Samuel, S. Phenomenological Gravitational Constraints on Strings and Higher Dimensional Theories. Phys. Rev. Lett. 1989, 63, 224.

10. Bluhm, R.; Kostelecký, V.A. Spontaneous Lorentz violation, Nambu-Goldstone modes, and gravity. Phys. Rev. D 2005, 71, 065008 .

11. Bluhm, R.; Fung, S.-H.; Kostelecký, V.A. Spontaneous Lorentz and diffeomorphism violation, massive modes, and gravity. Phys. Rev. D 2008, 77, 065020.

12. Bluhm, R.; Gagne, N.L.; Potting, R.; Vrublevskis, A. Constraints and stability in vector theories with spontaneous Lorentz violation. Phys. Rev. D 2008, 77, 125007.

13. Seifert, M.D. Vector models of gravitational Lorentz symmetry breaking. Phys. Rev. D 2009, 79, 124012.

14. Seifert, M.D. Generalized bumblebee models and Lorentz-violating electrodynamics. Phys. Rev. D 2010, $81,065010$.

15. Hernaski, C.A. Quantization and stability of bumblebee electrodynamics. Phys. Rev. D 2014, 90, 124036.

16. Escobar, C.A.; Urrutia, L.F. Photons emerging as Goldstone bosons from spontaneous Lorentz symmetry breaking: The Abelian Nambu model. Phys. Rev. D 2015, 92, 025042.

17. Jacobson, T.; Mattingly, D. Einstein-Aether Waves. Phys. Rev. D 2004, 70, 024003.

18. Jacobson, T. Einstein-aether gravity: A status report. 2007. Available online: https://arxiv.org/abs/0801.1547 (accessed on 9 March 2008).

19. Kostelecký, V.A.; Potting, R. Gravity from local Lorentz violation. Gen. Rel. Grav. 2005, 37, 1675.

20. Kostelecký, V.A.; Potting, R. Gravity from spontaneous Lorentz violation. Kostelecký, V.A.; Potting, R. Phys. Rev. D 2009, 79, 065018.

21. Altschul, B.; Bailey, Q.G.; Kostelecký, V.A. Lorentz violation with an antisymmetric tensor. Phys. Rev. D 2010, $81,065028$.

22. Hernaski, C. Spontaneous Breaking of Lorentz Symmetry with an antisymmetric tensor. Phys. Rev. D 2016, 94, 105004.

23. Jackiw, R.; Pi, S.-Y. Chern-Simons modification of general relativity. Phys. Rev. D 2003, 68, 104012.

24. Jackiw, R. Lorentz violation in a diffeomorphism-invariant theory. In CPT and Lorentz Symmetry IV; Kostelecký, V.A., Ed.; World Scientific: Singapore, 2008.

25. Hinterbichler, K. Theoretical Aspects of Massive Gravity. Rev. Mod. Phys. 2012, 84, 671. 
26. De Rham, C. Massive Gravity. Living Rev. Relativ. 2014, 17, 7.

27. Kostelecký, V.A.; Lehnert, R.; Perry, M.J. Spacetime-varying couplings and Lorentz violation. Phys. Rev. D 2003, 68, 123511.

28. Bertolami, O.; Lehnert, R.; Potting, R.; Ribeiro, A. Cosmological acceleration, varying couplings, and Lorentz breaking. Phys. Rev. D 2004, 69, 083513.

29. Ginges, J.S.M.; Flambaum, V.V. Violations of fundamental symmetries in atoms and tests of unification theories of elementary particles. Phys. Rept. 2004, 397, 63.

30. Uzan, J.-P. Varying Constants, Gravitation and Cosmology. Living Rev. Relativ. 2011, 14, 2.

31. Solà, J. Fundamental Constants in Physics and Their Time Variation. Mod. Phys. Lett. A 2015, 30, 1502004.

32. Bluhm, R. Spacetime symmetry breaking and Einstein-Maxwell theory. Phys. Rev. D 2015, 92, 085015.

33. Bluhm, R.; Šehić, A. Noether identities in gravity theories with nondynamical backgrounds and explicit spacetime symmetry breaking. Phys. Rev. D 2016, 94, 104034.

34. For reviews of experimental and theoretical approaches to violations of fundamental spacetime symmetries. In CPT and Lorentz Symmetry VII; Kostelecký, V.A., Ed.; World Scientific: Singapore, 2016.

35. Mattingly, D. Modern tests of Lorentz invariance. Living Rev. Relativ. 2005, 8, 5.

36. Mattingly, D.; Liberati, S. Lorentz breaking effective field theory models for matter and gravity: Theory and observational constraints. 2012. Available online: https:/ / arxiv.org/abs/1208.1071 (accessed on 6 August 2012).

37. Tasson, J. What do We Know about Lorentz Invariance? Rept. Prog. Phys. 2014, 77, 062901.

38. Bluhm, R. Observable Constraints on Local Lorentz Invariance. In Springer Handbook of Spacetime; Ashtekar, A., Petkov, V., Eds.; Springer: Berlin, Germany, 2014.

39. Iorio, L. Editorial for the Special Issue 100 Years of Chronogeometrodynamics: The Status of the Einstein's Theory of Gravitation in Its Centennial Year. Universe 2015, 1, 38.

40. Will, C.M. The Confrontation between General Relativity and Experiment. Living Rev. Relativ. 2014, 17, 4.

41. Hees, A.; Bailey, Q.G.; Bourgoin, A.; Bars, H.P.; Guerlin, C.; le Poncin-Lafitte, C. Tests of Lorentz symmetry in the gravitational sector. Universe 2016, 2, 30.

42. Debono, I.; Smoot, G.F. General Relativity and Cosmology: Unsolved Questions and Future Directions. Universe 2016, 2, 23.

43. Battat, J.B.R.; Chandler, J.F.; Stubbs, C.W. Testing for Lorentz Violation: Constraints on Standard-Model Extension Parameters via Lunar Laser Ranging. Phys. Rev. Lett. 2007, 99, 241103.

44. Mueller, H.; Chiow, S.-W.; Herrmann, S.; Chu, S.; Chung, K.-Y. Atom Interferometry tests of the isotropy of post-Newtonian gravity. Phys. Rev. Lett. 2008, 100, 031101.

45. Chung, K.-Y.; Chiow, S.-W.; Herrmann, S.; Chu, S.; Mueller, H. Atom interferometry tests of local Lorentz invariance in gravity and electrodynamics. Phys. Rev D 2009, 80, 016002.

46. Iorio, L. Orbital effects of Lorentz-violating Standard Model Extension gravitomagnetism around a static body: A sensitivity analysis. Class. Quant. Gravit. 2012, 29, 175007.

47. Yagi, K.; Blas, D.; Yunes, N.; Barausse, E. Strong Binary Pulsar Constraints on Lorentz Violation in Gravity. Phys. Rev. Lett. 2014, 112, 161101.

48. Shao, L. Tests of local Lorentz invariance violation of gravity in the standard model extension with pulsars. Phys. Rev. Lett. 2014, 112, 111103.

49. Shao, L. New pulsar limit on local Lorentz invariance violation of gravity in the standard-model extension. Phys. Rev D 2014, 90, 122009.

50. Bourgoin, A.; Hees, A.; Bouquillon, S.; le Poncin-Lafitte, C.; Francou, G.; Angonin, M.-C. Testing Lorentz symmetry with Lunar Laser Ranging. Phys. Rev. Lett. 2016, 117, 241301.

51. Bourgoin, A.; le Poncin-Lafitte, C.; Hees, A.; Bouquillon, S.; Francou, G.; Angonin, M.-C. Lorentz symmetry violations from matter-gravity couplings with Lunar Laser Ranging. 2017. Available online: https:/ /arxiv. org/abs / 1706.06294 (accessed on 20 June 2017).

52. Kostelecký, V.A.; Russell, N. Data tables for Lorentz and CPT violation. Rev. Mod. Phys. 2011, 83, 11.

53. Bailey, Q.G.; Kostelecký, V.A. Signals for Lorentz violation in post-Newtonian gravity. Phys. Rev. D 2006, $74,045001$.

54. Kostelecký, V.A.; Tasson, J.D. Prospects for Large Relativity Violations in Matter-Gravity Couplings. Phys. Rev. Lett. 2009, 102, 010402.

55. Kostelecký, V.A.; Tasson, J.D. Matter-gravity couplings and Lorentz violation. Phys. Rev. D 2011, $83,016013$. 
56. Kostelecký, V.A.; Tasson, J.D. Constraints on Lorentz violation from gravitational Cherenkov radiation. Phys. Lett. B 2015, 749, 551.

57. Hernaski, C.A.; Belich, H. Lorentz violation and higher-derivative gravity. Phys. Rev. D 2014, 89, 104027.

58. Bailey, Q.G.; Kostelecký, V.A.; Xu, R. Short-range gravity and Lorentz violation. Phys. Rev. D 2015, 91, 022006.

59. Lane, C.D. Spacetime variation of Lorentz-violation coefficients at a nonrelativistic scale. Phys. Rev. D 2016, 94, 025016.

60. Shao, C.-G.; Tan, Y.-J.; Tan, W.-H.; Yang, S.-Q.; Luo, J.; Tobar, M.E.; Bailey, Q.G.; Long, J.C.; Weisman, E.; $\mathrm{Xu}, \mathrm{R} . ;$

61. Kostelecký, V.A.; Mewes, M. Testing local Lorentz invariance with gravitational waves. Phys. Lett. B 2016, 757,510 .

62. Testing local Lorentz invariance with short-range gravity. Phys. Lett. B 2017, 766, 137.

63. Kostelecký, V.A.; Melissinos, A.C.; Mewes, M. Searching for photon-sector Lorentz violation using gravitational-wave detectors. Phys. Lett. B 2016, 761, 1.

64. Arkani-Hamed, N.; Georgi, H.; Schwartz, M.D. Effective field theory for massive gravitons and gravity in theory space. Ann. Phys. 2003, 305, 96.

65. Bjorken, J.D. A Dynamical origin for the electromagnetic field. Ann. Phys. 1963, 24, 174.

66. Nambu, Y. Quantum electrodynamics in nonlinear gauge. Prog. Theor. Phys. Suppl. E 1968, 68, 190.

67. Noether, E. Invariante Variationsprobleme (Invariant Variation Problems). Nachr. D. König. Gesellsch. D. Wiss. Göttingen: Math-Phys. Klasse 1918, 1918, 235-257. (In German)

68. Trautman, A. Conservation laws in general relativity. In Gravitation: An Introduction to Current Research; Witten, L., Ed.; J. Wiley: New York, NY, USA, 1962.

(C) 2017 by the author. Licensee MDPI, Basel, Switzerland. This article is an open access article distributed under the terms and conditions of the Creative Commons Attribution (CC BY) license (http://creativecommons.org/licenses/by/4.0/). 